\title{
Intelligent Risk Evaluation of Transmission Line Icing Based on Bayesian ANFIS
}

\author{
HUANG Yali, ${ }^{1,}$, MA Tiannan ${ }^{1, b}$, WU Meiqiong ${ }^{1, c}$ and NIU Dongxiao ${ }^{1, d}$ \\ ${ }^{1}$ Research Institute of Technology Economics Forecasting and Assessment, \\ North China Electric Power University, Beijing 102206, China

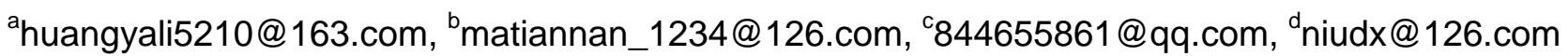

\begin{abstract}
Keywords: Bayesian inference; Adaptive Network-based Fuzzy Inference Systems (ANFIS); Icing risk evaluation

Abstract. Transmission line icing is a serious threat to the security of power system. Risk evaluation for icing is the foundation of building ice disaster defense system and ensuring the safety of power system. In this paper, the index system of transmission line icing risk assessment was developed in consideration of the influence factors. By applying Bayesian inference to ANFIS, the Bayesian ANFIS icing risk evaluation model was established and was applied into practice. Experiments showed that, the ANFIS system can optimize the parameters of the network by applying Bayesian inference and has good learning abilities and adaptabilities. The method is effective and feasible in the risk evaluation of transmission line icing.
\end{abstract}

\section{Introduction}

With the high frequency of disastrous weather such as global continuous low temperature, rain, snow, ice, haze and fog, the grid disasters are aggravating. The damage caused by transmission line icing becomes more and more severe. Freeze disaster has become a significant problem of the grid system [1], seriously threatening the safe operation of power system [2]. The risk evaluation of icing, line trip and insulator flashover is the foundation of establishing ice disaster defense system. In order to reduce the lines icing accidents and effectively guarantee the safe operation of the power system, it is necessary to establish on-line monitoring and warning system of line icing to report the possible freeze disaster risks timely and accurately. So it becomes essential to carry out transmission line icing risk evaluation.

At present, there are mainly three aspects of the research related to transmission line icing risk evaluation. The first is the research about the reliability of the power grid operation under freezing weather conditions. In the research, the focuses are mainly on the states of the transmission components and transmission network failure modes under different climate conditions. Besides, the calculation formulas of system failure rate and average interruption duration have been derived [3-5]. This aspect is the study of the transmission network during the freezing disasters, with the lack of analysis before the freeze disaster. The second is devoted to the study of the risk assessment of transmission line icing. There are a few such studies currently. And the majority of the literatures still focus on the cost and the economy of the transmission line icing [6], the power system security assessment framework [7], the safety assessment under extreme snow and ice disaster [8], and so on, which lacks a scientific, practical and comprehensive icing risk assessment model. The third is the research of on-line monitoring of transmission lines. Online monitoring can contribute to the on-line analysis of line icing state and predicting the safety status of electrical equipment, which is the basis of warning grid faults and maintaining the safe and stable operation of power grids. Japanese Koichi Nara and other men established the overhead line icing prevention decision-making expert system for $187 \mathrm{kV}$ and $66 \mathrm{kV}$, using the on-line meteorological data such as temperature to judge the current state of overhead lines. So the operators can choose how to deal with the line icing, to prevent or to get rid of the ice [9]. But the correlative work about the on-line icing risk assessment is seldom inland.

From the above, we know that currently there is a lack of scientific, practical and comprehensive icing risk assessment model to guide the design of transmission lines and the icing emergency 
management. And the study about the on-line transmission line icing risk assessment is almost blank particularly. Therefore, the full use of online monitoring data was made and the intelligent evaluation model was combined effectively to evaluate the transmission line icing risk systematically.

Adaptive Network-based Fuzzy Inference Systems (ANFIS) is the a combination of fuzzy inference and neural networks, which not only has the adaptability and learning ability of neural network, but also enables the nodes and weights of the neural network to have definitude physical meanings. Statistical inference is based on the posterior distribution in Bayesian inference. ANFIS based on Bayesian inference can adaptively tweak the regularization parameters to achieve the optimal ones by focusing on the probability distribution of the whole parameter space during training the neural network. Meanwhile, Bayesian ANFIS has more robust and better generalization capacity in theory [10].

In this paper, transmission line icing risk evaluation index system was established and icing risk level was divided based on the transmission line on-line monitoring data and the comprehensive analysis of various icing influence factors such as temperature, humidity, wind speed. Then the intelligent Bayesian ANFIS line icing risk evaluation model was obtained by applying Bayesian inference to optimize the parameters of ANFIS model. Finally, adapt this model for the regional power grid.

\section{Bayesian ANFIS Icing Risk Evaluation Model}

Basic ANFIS.ANFIS is a system which can be built by self-adaptive modeling based on a large sample of data [11]. It assumes a parameterized fuzzy structure firstly and uses the data to train the Fuzzy Inference System (FIS) model. Then, according to a selected error criterion, it corrects the member function parameters to make the FIS harmonize the training data [12-13]. ANFIS can get rid of the condition where the membership functions are designed by relying on the human mind in the process of the traditional fuzzy logic reasoning. So it can reduce the error and increasing the system efficiency. ANFIS automatically adjusts its variable membership function parameters continually to make itself adapt to the best of input and output relationships by self-training and self-learning on the basis of the established fuzzy rules.

Bayesian ANFIS. Regarding the experience and knowledge as a priori information, Bayesian inference amends the previous knowledge and the previous distribution continually combined with the current samples. So the parameters are more and more precise [10]. The following formula can be used.

$$
P(\theta / x)=\frac{P(x / \theta) P(\theta)}{\int_{\theta} P(x / \theta) P(\theta) d \theta}
$$

Where $P(\theta / x)$ indicates the modified probability value after sampling. $P(\theta)$ indicates cognitive probability value before sampling. $P(\theta / x)$ refers to the result of the adjustment of $P(\theta)$.

The objective function in basic ANFIS is usually the likelihood function of the data and the regularization corresponds to the priori probability distribution when applying Bayesian inference to ANFIS. And all the parameters of the network are regarded as random variables. Then it learns in the whole weight space and gets the posterior conditional probability of the parameters based on the assumption of prior probability distribution. Finally, it obtains the optimal parameters values based on the posterior distribution of Bayesian inference [10].

The difference between Bayesian ANFIS and basic ANFIS is that Bayesian ANFIS focuses on the probability distribution in the whole parameter space and predicts based on the average of the posterior probability distribution. One model corresponds to a point in the parameter space and all models correspond to the whole parameter space. So Bayesian ANFIS has more generalization ability in theory [10].

In general, the error of the typical feed-forward neural network is mean square error. Here $E_{D}$ is computed by 


$$
E_{D}=\frac{1}{N} \sum_{i=1}^{N}\left(e_{i}\right)^{2}
$$

Where $\mathrm{N}$ is the total number of the samples, $e_{i}$ is the error.

In order to modify the error function, the mean square errors of the network weights and the thresholds are added [10].

$$
\begin{gathered}
F=\beta E_{D}+\alpha E_{W} \\
E_{W}=\frac{1}{N} \sum_{i=1}^{N}\left(w_{i}\right)
\end{gathered}
$$

Where $\alpha$ and $\beta$ are regularization parameters. $W_{i}$ is network weight.

The weights and thresholds can be smaller by applying the formula (8) to modify the error function, which means to make the effective weights and thresholds as small as possible. This is actually automatically reducing the size of the network, so as to make the network response smoother and reduce over training.

$\alpha$ and $\beta$ are named as super parameters, controlling the distribution of other parameters (weights and thresholds). The size of the super parameter determines the training targets of the neural network [10]. If $\alpha \leqslant \beta$, the training target is to reduce the training error of the network. If $\alpha \geqslant \beta$, the training aims at making the network response smoother, which means to reduce the number of the effective network parameters as possible to make up the large network error. In a practical application, this objective needs tradeoffs and minimizing is to reduce the complexity of the network structure when reducing the network training error [10]. The conventional regularization methods are always difficult to determine the size of the regularization parameters. But Bayesian ANFIS can adaptively adjust the size of the regularization parameters in the process of network training and make them optimal [10].

Regard $\alpha$ and $\beta$ as the random variables. Based on Bayesian theory, their posterior distributions are computed by

$$
P(\alpha, \beta / D, M)=\frac{P(D / \alpha, \beta, M) P(\alpha, \beta / M)}{P(D / M)}
$$

Assume $P(\alpha, \beta / M)$ is a wide distribution. Because it is irrelevant to the normalization factor, $P(D / M)$, it is called the significant degree of $\alpha$ and $\beta$. Therefore, to make the posterior distribution of $\alpha$ and $\beta$ maximum, the only need is to make the likelihood function, $P(D / \alpha, \beta, M)$, maximum [10].

Bayesian focuses on the probability distribution of the weight (threshold) in the whole space. Use $\mathrm{M}$ to represent the network structure, which means the number of hidden layer neurons. Under the condition of the network structure determined, the prior distribution of the weight (the threshold) is $P(w / \alpha, M)$ without any samples. Where $\mathrm{w}$ is the weight (the threshold) vector. And the posterior distribution is $P(w / D, \alpha, \beta, M)$ with the sample date sets, D. Based on the Bayesian, $P(w / D, \alpha, \beta, M)$ is computed by

$$
P(w / D, \alpha, \beta, M)=\frac{P(D / w, \beta, M) P(w / \alpha, M)}{P(D / \alpha, \beta, M)}
$$

The prior distribution is a wide distribution due to low awareness of the distribution of the weight without any data, while the posterior distribution is compact with data. That is, only the weight in a very small range may be in line with the network mapping. In order to obtain the posterior distribution, the prior distribution and the likelihood function should be known at first.

According to the literature [10], the prior distribution of the network weight obeys Gauss distribution. 


\section{Transmission Line Icing Risk Evaluation Index System}

Transmission Line Icing Risk Evaluation Index System.The main factors of transmission line icing can be divided into three groups: meteorological factors, environmental factors and line parameters.

Different conditions of the factors determine the different types, degree and the risk levels of icing. According to the factors, the icing risk evaluation index system is established, as shown in Table 1 [14].

Table 1 Icing Risk Evaluation Index System

\begin{tabular}{c|c|cc}
\hline & First class indexes & Second class indexes & \\
\hline & & Temperature & $\mathrm{X}_{11}$ \\
& \multirow{3}{*}{ Meteorological factors } & Humidity & $\mathrm{X}_{12}$ \\
& & Wind speed & $\mathrm{X}_{13}$ \\
\cline { 2 - 4 } Icing Risk & \multirow{3}{*}{ Environmental factors } & Wind direction & $\mathrm{X}_{14}$ \\
\cline { 2 - 4 } System & & Altitude & $\mathrm{X}_{21}$ \\
& & Terrain & $\mathrm{X}_{22}$ \\
& & Forest & $\mathrm{X}_{23}$ \\
\cline { 2 - 4 } & \multirow{2}{*}{ Line parameters } & Line route & $\mathrm{X}_{31}$ \\
& & Conductor suspension height & $\mathrm{X}_{32}$ \\
& & Wire rigidity & $\mathrm{X}_{33}$ \\
& & Wire diameter & $\mathrm{X}_{34}$ \\
& & Load current & $\mathrm{X}_{35}$ \\
\hline
\end{tabular}

Transmission Line Icing Risk Level.In this paper, the line icing thickness is adopted as the standard of the line icing risk level, as shown in Table 2 [15].

\begin{tabular}{c|c|c}
\multicolumn{3}{c}{ Table 2 Icing Risk Level } \\
\hline $\begin{array}{c}\text { Icing } \\
\text { thickness } \\
(\mathrm{mm})\end{array}$ & Icing risk level & $\begin{array}{c}\text { Degree of } \\
\text { icing risk }\end{array}$ \\
\hline 0 & 1 & None \\
$0 \sim 10$ & 2 & Light \\
$10 \sim 20$ & 3 & Medium \\
$20 \sim 30$ & 4 & Serious \\
30 above & 5 & Super serious \\
\hline
\end{tabular}

The icing thickness is the difference the wire icing diameter (the data reported from observation station) and the wine diameter and shown in millimeter.

\section{Empirical Analysis}

The data monitored at Zhongkuang Line No. 55 in Hunan Province during January and February in 2015 were selected in this paper. In order to ensure the comprehensiveness of all the variables, 126 samples were screened. And 106 of them were used as training samples, accounting for $84.1 \%$ of the total samples, while 20 samples as testing samples, accounting for $15.9 \%$. Besides, the temperature, humidity, wind speed and icing thickness of all the samples are shown in Figure 1.

Firstly, the original data were normalized by

$$
x_{\mathrm{n}}=\frac{x-\frac{x_{\max }+x_{\min }}{2}}{\frac{x_{\max }-x_{\min }}{2}}
$$

Where $x_{\mathrm{n}}$ is the normalized. $x$ is the original. $x_{\max }$ is the maximum of all and $x_{\min }$ is the minimum of all. 
The relative error (RE), the root mean square error (RMSE) and the mean absolute percentage error (MAPE) were also adopted to evaluate the results of the algorithm.

Then, in order to make a comparison, the basic ANFIS model and the BP neural network model were always applied to this paper to test the data above. The parameter values were set as follow.
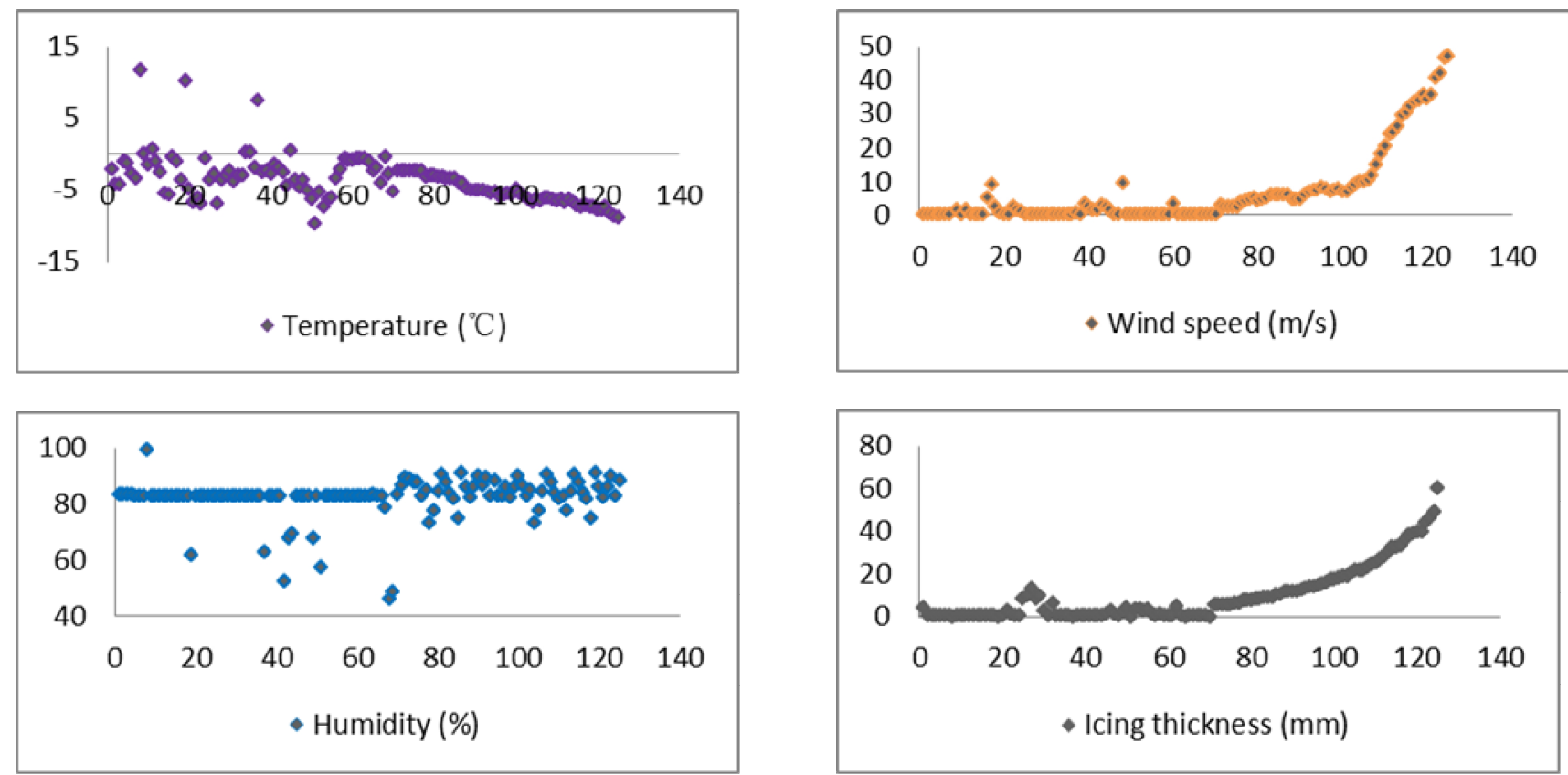

Figure 1 The Temperature, Humidity, Wind speed and Icing thickness of All Samples

(1) Bayesian ANFIS

The ranges of input variable $\mathrm{x}$ and output variable $\mathrm{y}$ were both [-1,1]. Each variable only took 2 values in the fuzzy layer on the premise of meeting certain accuracy, so 256 rules were set. The generalized bell-type membership function was adopted as input fuzzy membership function because it can reflect the reality well. And the hybrid algorithm was chosen in the Bayesian ANFIS training, while the error accuracy was set to 0.001 , the training number was 500 .

\section{(2)Basic ANFIS}

The parameter values were set as the Bayesian ANFIS in basic ANFIS. The ranges of input variable $\mathrm{x}$ and output variable $\mathrm{y}$ were both $[-1,1]$. Each variable only took 2 values in the fuzzy layer on the premise of meeting certain accuracy, so 256 rules were set. The generalized bell-type membership function was adopted as input fuzzy membership function. And the hybrid algorithm was chosen in the basic ANFIS training, while the error accuracy was set to 0.001, the training number was 500 .

(3) BP neural network

The numbers of node in input layer, hidden layer and output layer of BP neural network were 12, 8 , 1. The maximum training times was 500; the learning rate was 0.01 ; the precision was 0.001 ; the frequency was 20 ; the additional momentum factor was 0.95 ; the minimum performance gradient was 1e-6; the maximum failure number was 5. And the traingd function was chosen as training function.

Next, the normalized data were input to the various models above to get the results by MATLAB, as shown in Table 4.

Figure 2 is the training iteration root mean square error of Bayesian ANFIS, basic ANFIS and BP neural network. From the figure, the iterations of Bayesian ANFIS, basic ANFIS and BP neural network stopped at 124 times, 201 times and 222 times, while the error precision reached 0.00092, 0.00099 and $0.00097(<0.001)$. It can be seen that the Bayesian ANFIS algorithm can converge quickly and meet the expected results in a short time. It also can be inferred that the nonlinear fitting ability of the ANFIS algorithm was improved by optimizing the weights based on Bayesian inference, so as the generalization ability. 


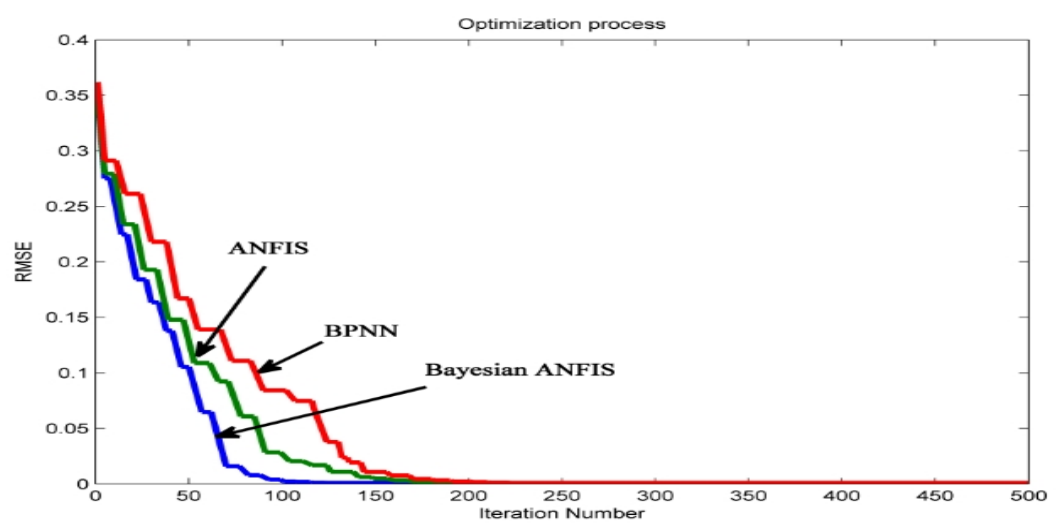

Figure 2 The Training Error Iteration of Each Algorithm

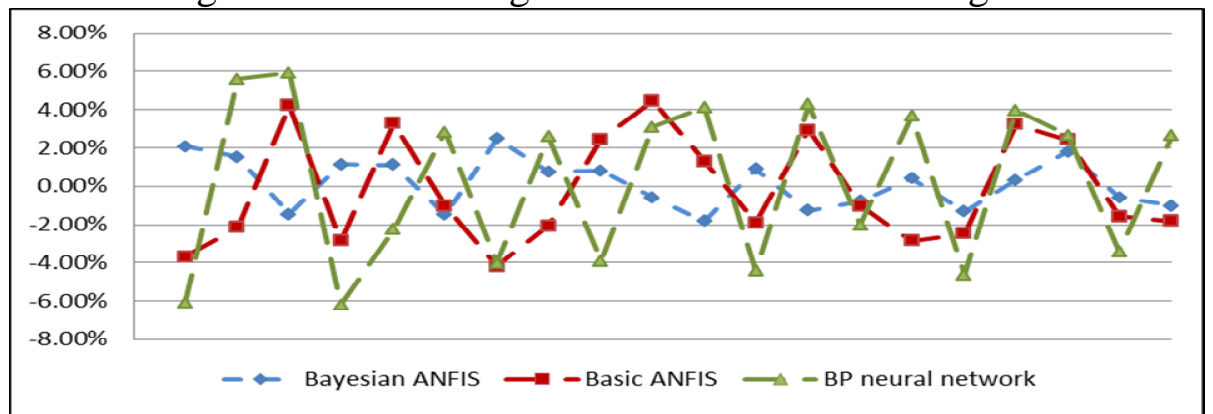

Figure 3 The Relative Error of Each Algorithm

Table 3 The Comparison of the Predicting Values and the True Values

\begin{tabular}{|c|c|c|c|c|c|c|c|c|c|}
\hline \multirow[b]{2}{*}{$\begin{array}{l}\text { Testing } \\
\text { samples }\end{array}$} & \multirow[b]{2}{*}{$\begin{array}{l}\text { True } \\
\text { value }\end{array}$} & \multirow[b]{2}{*}{$\begin{array}{c}\text { Actual } \\
\text { risk } \\
\text { level }\end{array}$} & \multirow{2}{*}{$\begin{array}{c}\text { Risk } \\
\text { level } \\
\text { evaluated } \\
\text { by } \\
\text { Bayesian } \\
\text { ANFIS } \\
\end{array}$} & \multicolumn{2}{|c|}{ Bayesian ANFIS } & \multicolumn{2}{|c|}{ Basic ANFIS } & \multicolumn{2}{|c|}{ BP neural network } \\
\hline & & & & $\begin{array}{l}\text { Predicting } \\
\text { value }\end{array}$ & $\begin{array}{c}\text { Relative } \\
\text { error }\end{array}$ & $\begin{array}{l}\text { Predicting } \\
\text { value }\end{array}$ & $\begin{array}{l}\text { Relative } \\
\text { error }\end{array}$ & $\begin{array}{l}\text { Predicting } \\
\text { value }\end{array}$ & $\begin{array}{c}\text { Relative } \\
\text { error }\end{array}$ \\
\hline 1 & 0.59 & 2 & 2 & 0.60 & $2.07 \%$ & 0.56 & $-3.69 \%$ & 0.55 & $-6.11 \%$ \\
\hline 2 & 5.15 & 2 & 2 & 5.23 & $1.53 \%$ & 5.04 & $-2.15 \%$ & 5.44 & $5.59 \%$ \\
\hline 3 & 5.27 & 2 & 2 & 5.19 & $-1.47 \%$ & 5.49 & $4.21 \%$ & 5.58 & $5.91 \%$ \\
\hline 4 & 6.51 & 2 & 2 & 6.59 & $1.15 \%$ & 6.33 & $-2.87 \%$ & 6.11 & $-6.20 \%$ \\
\hline 5 & 7.40 & 2 & 2 & 7.48 & $1.12 \%$ & 7.64 & $3.28 \%$ & 7.23 & $-2.26 \%$ \\
\hline 6 & 8.19 & 2 & 2 & 8.07 & $-1.51 \%$ & 8.11 & $-1.04 \%$ & 8.42 & $2.82 \%$ \\
\hline 7 & 8.89 & 2 & 2 & 9.11 & $2.47 \%$ & 8.52 & $-4.18 \%$ & 8.53 & $-4.03 \%$ \\
\hline 8 & 10.20 & 3 & 3 & 10.28 & $0.77 \%$ & 9.99 & $-2.06 \%$ & 10.47 & $2.59 \%$ \\
\hline 9 & 11.70 & 3 & 3 & 11.80 & $0.82 \%$ & 11.99 & $2.44 \%$ & 11.25 & $-3.92 \%$ \\
\hline 10 & 13.50 & 3 & 3 & 13.42 & $-0.60 \%$ & 14.10 & $4.46 \%$ & 13.92 & $3.09 \%$ \\
\hline 11 & 14.80 & 3 & 3 & 14.53 & $-1.83 \%$ & 14.99 & $1.31 \%$ & 15.41 & $4.14 \%$ \\
\hline 12 & 15.35 & 3 & 3 & 15.49 & $0.91 \%$ & 15.06 & $-1.91 \%$ & 14.67 & $-4.44 \%$ \\
\hline 13 & 17.78 & 3 & 3 & 17.55 & $-1.29 \%$ & 18.30 & $2.93 \%$ & 18.54 & $4.28 \%$ \\
\hline 14 & 18.40 & 3 & 3 & 18.25 & $-0.80 \%$ & 18.21 & $-1.04 \%$ & 18.03 & $-2.02 \%$ \\
\hline 15 & 22.03 & 4 & 4 & 22.12 & $0.41 \%$ & 21.40 & $-2.85 \%$ & 22.84 & $3.68 \%$ \\
\hline 16 & 32.51 & 5 & 5 & 32.08 & $-1.34 \%$ & 31.71 & $-2.46 \%$ & 31.01 & $-4.63 \%$ \\
\hline 17 & 33.58 & 5 & 5 & 33.69 & $0.32 \%$ & 34.67 & $3.24 \%$ & 34.91 & $3.96 \%$ \\
\hline 18 & 38.19 & 5 & 5 & 38.88 & $1.80 \%$ & 39.12 & $2.43 \%$ & 39.21 & $2.66 \%$ \\
\hline 19 & 39.89 & 5 & 5 & 39.65 & $-0.60 \%$ & 39.27 & $-1.57 \%$ & 38.52 & $-3.44 \%$ \\
\hline 20 & 49.20 & 5 & 5 & 48.70 & $-1.03 \%$ & 48.31 & $-1.82 \%$ & 50.50 & $2.62 \%$ \\
\hline RMSE & & & & & $1.31 \%$ & & $2.78 \%$ & & $4.11 \%$ \\
\hline MAPE & & & & & $1.19 \%$ & & $2.60 \%$ & & $3.92 \%$ \\
\hline
\end{tabular}


Table 3 is the prediction results of the 3 algorithms. Figure 3 is the testing error of the 3 algorithms. Compare the testing results of Bayesian ANFIS, basic ANFIS and BP neural network and it can be seen that the maximum gap between the predicting value of Bayesian ANFIS and the actual value is $0.69 \mathrm{~mm}$, which is less than the maximum gap of basic ANFIS, $1.09 \mathrm{~mm}$ and the maximum gap of BP neural network, $1.50 \mathrm{~mm}$. And the minimum gap between the predicting value of Bayesian ANFIS and the actual value is $0.01 \mathrm{~mm}$, which is also less than that of basic ANFIS, $0.02 \mathrm{~mm}$ and that of BP neural network, $0.04 \mathrm{~mm}$. It shows that the Bayesian ANFIS model has more stable predicting results than the basic ANFIS model and the BP neural network model.

The maximum values of the absolute relative error of Bayesian ANFIS, basic ANFIS and BP neural network were $2.47 \%, 4.46 \%$, and $6.20 \%$, while the minimum value were $0.32 \%, 1.04 \%, 2.02 \%$. It showed that the Bayesian ANFIS model had higher accuracy, more accurate predictions and more robustness. The root mean square error is to measure the variation between the predicting values and the true values. As seen in Table 4, the root mean square error values of Bayesian ANFIS, basic ANFIS and BP neural network were $1.31 \%, 2.78 \%$, and $4.11 \%$, which indicated that the difference between the predicting value of the Bayesian ANFIS model and the true value was the least. So the Bayesian ANFIS model obtained the best prediction effect and the basic ANFIS model, the BP neural network model follow in turn. In addition, it can be seen that the mean absolute percentage error values of the Bayesian ANFIS algorithm was $1.19 \%$, which was less than that of the basic ANFIS algorithm, $2.60 \%$, and $3.92 \%$ of the BP neural network algorithm. It indicated that the Bayesian ANFIS model had the highest overall prediction accuracy and the strongest nonlinearity mapping ability and was more suitable for the evaluation of transmission line icing risk.

In the icing risk evaluation results of Bayesian ANFIS, there were 7 samples in the risk level 2 and they were samples 1-7; there were also 7 samples in the risk level 3 and they were samples 8-14; there was only one sample in the risk level 4 and it was sample 15; there were 5 samples in the risk level 5 and they were samples 16-20. From the results, the accuracy of transmission line icing risk evaluation by Bayesian ANFIS was 100\%.

\section{Conclusions}

First, the transmission line icing risk evaluation index system was established according to the influence factors of icing in this paper. Then the Bayesian ANFIS icing risk evaluation model was built by applying Bayesian inference to the ANFIS algorithm to evaluate the risk of transmission line icing. This paper selected Hunan Province line, Zhongkuang line 55, as the case to test and verify the Bayesian ANFIS model. The results showed that Bayesian ANFIS system could optimize the network parameters continually and obtained good learning ability and adaptability. And the comparison with the basic ANFIS algorithm and the BP neural network algorithm indicated that the Bayesian ANFIS model had a higher prediction accuracy, stronger robustness and better evaluation effect of the transmission line icing risk.

\section{Acknowledgements}

This work was financially supported by The National Natural Science Foundation of China (71471059) and the Fundamental Research Funds for the Central Universities (Grant no. 2015XS36).

\section{References}

[1] Masoud Farzaneh. Atmospheric Icing of Power Networks[M]. Springer, 2008.

[2] Chengrong Li, Yuzhen Lv, Xiang Cui, et al. Research issues for safe operation of power grid in China under ice-snow disasters[J]. Power System Technology, 2008, 32(4): 14-21.

[3] Yang Liu, Jiaqi Zhou. Incorporating weather effect in bulk power system reliability evaluation[J]. Electric Power Automation Equipment, 2003, 23(9): 60-62. 
[4] Ming Ding, Renchang Dai, Mei Hong, Xianyong Xu. Simulation to the weather condition affecting the reliability of transmission network[J]. Automation of Electric Power Systems, 1997, 21(1): 18-20.

[5] Yongjin Chen, Zhen Ren, Wenying Huang. Model and analysis of power system reliability evaluation considering weather change[J]. Automation of Electric Power Systems, 2004, 28(21): 17-21.

[6] Yongjun Zhang, Hui Huang, Jie Tang. Economy comparison of line's ice disaster cycle based on non-sequential Monte Carlo[J]. Power System Protection and Control, 2010, 38(13): 119-123.

[7] Rongfu Sun, Lin Cheng, Yuanzhang Sun. An outage rate model and system adequacy assessment based on adverse weather conditions[J]. Automation of Electric Power Systems, 2009, 33(13): 7-13

[8] Hengxu Zhang, Yutian Liu, Pengfei Zhang. Requirements analysis and framework design for power system security assessment considering extreme ice disasters[J]. Proceedings of the CSEE, 2009, 29(16): 8-14.

[9] Nara K, Yamashiro S, Yanaura Y. Application of an Expert System to Decisions on Countermeasures Against Snow Accretion on Transmission Lines[J]. IEEE Transactions on Power Systems, 1988, 3(3): 1052-1058.

[10]Peng Li. Research of Neural Network Algorithm based on Bayes Theory[J]. Ome Information, 2011, 01: 28-32.

[11] Jiangliao Wu, Lingzhi Yi, Hanmei Peng, Xiang Liu, Wenlang Deng. Position sensorless control of the switched reluctance motor using adaptive network fuzzy inference system (ANFIS)[J]. Journal of China Coal Society, 2010, 08: 1401-1405.

[12] Pengcheng Li, Guohua Chen, Li Zhang, Licao Dai, Qingqing Chen. An ANFIS-based approach to identifying criticality of human error risk[J]. China Safety Science Journal, 2014, 01: 72-77.

[13] T. G. Ling, M. F. Rahmat, A. R. Husain. ANFIS Modeling and Direct ANFIS Inverse Control of an Electro-Hydraulic Actuator System[J]. 2013 IEEE 8th Conference on Industrial Electronics and Applications (ICIEA), 2013.

[14]Zehong Liu. Direct-voltage transmission line icing and prevention[M]. Beijing: China Electric Power Press, 2012: 9-19.

[15] Shuiqing Yin, Shanshan Zhao, Zunya Wang, Qiang Zhang, Weian Tang. Characteristic analysis of ice accumulation on transmission lines and simulation based on ANN model over China[J]. Journal of Applied Meteorological Science, 2009, 06: 722-728. 